\title{
CALIBRATION OF BTT MEASUREMENT WITH RESPECT TO SENSOR POSITION OVER SHROUDED LSB
}

\author{
Tereza DadÁKovÁ*, ZdenĚK KubíN \\ Doosan Škoda Power s.r.o., Tylova 1/57, Pilsen, Czech Republic \\ * corresponding author: tereza.dadakova@doosan.com
}

\begin{abstract}
In order to increase efficiency and reliability of steam turbines, last stage blades are equipped with mid-span tie-boss and shroud. We can use Blade Tip-Timing method (BTT) to measure vibration of shrouded blades, but the measured level of vibration is dependent on position of sensor over blade shroud. Hence, the results of BTT measurement have to be compared with other measurement techniques, like strain gauges, and with FEM analysis, to interpret these results right. This article provides findings we made on measurement of shrouded blades in test rig with eddy-current sensors, optical sensors and strain gauges at different positions of sensors over blade shroud. Measurement is also compared to FEM analysis.
\end{abstract}

KEywords: Blade Tip-Timing, calibration, sensor.

\section{INTRODUCTION}

Blade Tip Timing (BTT) measurement is in Doosan Skoda Power used mainly for two reasons: To prevent blade failure on some operational states, where can be increased level of vibration because of ventilation unpredictable asynchronous vibration can be excited by small aerodynamic forces. For these cases, longterm monitoring with calculation of blade lifetime is used on power plants to plan shutdowns of turbine and measuring blade behaviour on wide range of operation points to optimalize operation of the turbine. Second use of BTT in Doosan Skoda Power is blade testing in Campbell test rig to compare measurements with FEM Analysis and prove that the blades are designed out of synchronous resonances. For shrouded blades we use Campbell test rig to do the calibration of BTT - determination of suitable position for sensor location, comparison of results of BTT with strain gages and verification of calculation.

The need to do the calibration of BTT occurred with the new design of blades with interconnecting elements. To last stage blade (LSB) design tie-bos in the middle of the blade and shroud at the tip of the blade were added. The centrifugal force causes untwisting of blades, resulting in force between these contact elements and hence in reduction of blade vibration. However these features brought many questions about measuring those blades with BTT. The blade shroud has a great impact on signal shape from the sensor, in some positions (contact plane) it is complicated to receive a good data. Another question is what point of shroud is exactly measured and it is necessary to interpret the amplitude of vibration for BTT right.

Also the identification of cracks on shrouded blades became more demanding task. Cracks usually cause a change in blade vibration behaviour like frequency shift, higher level of vibration, shift of a blade towards other blades - change in inner blade distance, etc.
However on free blades is this behavior manifested more significantly.

An alternative measurement to BTT are contact methods - use of strain gages. Strain gages give us direct information about the blade stress, but usually are installed only on few blades and in corrosive steam enviroment with high temperature usually have a short service life. Also the installation is quite expensive and these reasons make this method unsuitable for wider use.

BTT as contactless method, or NSMS (Non- Intrusive Stress Measurement System), is cheaper, sensors are placed circumferentially around stator and hence each sensor sees each blade of the rotating disk [1]. The BTT system measures time arrival of each blade, the difference between time arrivals is converted to vibration in $\mathrm{mm}$. This data can be analyzed in many ways, using various methods 2 , 3. Most used method is Fast Fourier Transformation (FFT) converting the signal from a sensor to single-blade spectrums or allblade spectrum. Single-blade spectrum gives us information about vibration of every blade. However, it is sampled only once per revolution what causes limited frequency range and problems with identification of natural frequency and nodal diameter. On the other side, all-blade spectrum shows us the whole disk vibration behaviour with possibility of determination nodal diameters and natural frequencies (It is sampled by number of blades in one revolution), but changes in vibration of one blade with defect may not occur in this spectrum. This usually leads to using a combination of different methods to receive a real amplitude of all blades. Various types of sensors can be used, new sensors with different properties are developed [4. Sensors can be based on different physical principals, not in every case it is clear what exactly sensors measure. Simulation of measurement can help here $[5,6]$. 
This article contains observations based on measurement of shrouded blade in Campbell test rig, using optical sensor, eddy-current sensors and strain gages. This measurement was special, because of using a system that gradually moved sensors over rotating blades. This way we were able to "scan" blade shroud and find best sensor position for measuring this type of blade. It also gave us a different point of view on measuring with eddy-current sensors. We also describe a method we use to calibrate BTT with calculation.

\section{Measurement Description}

The measurement was done in Campbell test rig on LSB for $60 \mathrm{~Hz}$ with diameter $2904 \mathrm{~mm}$, the disk consisted of 50 blades. The Campbell test rig used for measurement operates without steam, blades rotate in vacuum, because high-speed motion through air would result in aerodynamic heating and rise of temperature. The level of vacuum is usually about $3 \mathrm{mbar}$, maximal rotational speed is $4000 \mathrm{RPM}$ and maximal disk diameter is $5 \mathrm{~m}$.

To excite blades an electro-magnet, oil jets or torsional shaking can be used. The electo-magnet excites blades on frequency $f_{e}$,

$$
f_{e}=f_{n}+N D \cdot f_{r},
$$

where $f_{n}$ is natural blade frequency, $N D$ is Nodal Diameter which is excited and $f_{r}$ is rotational frequency. Electro-motor torsional shaking is used to excite $0 N D$, because from our experience the amplitude is ten-times bigger, than by using the electromagnet. Unfortunately, only this nodal diameter can be excited this way.

\subsection{Measuring System}

To measure the blade vibration contact and contactless methods were used, as mentioned in section 1 . In the test rig it is possible to use strain gages with telemetry system, BTT and high speed camera monitoring system. For this measurement only strain gages and BTT were used.

Strain gages were placed at positions, where we expected maximal response to verify shapes of various nodal diameters. Amplitudes are obtained from Fast Fourier Transformation directly on natural frequency.

BTT consists of sensor, amplifier, trigger and measuring system with high sampling frequency clock (Figure 1).

In the test rig we successfully use optical sensors, but in steam enviroment the use of optical sensors is problematic. For use in steam enviroment eddycurrent sensors are more suitable, because they are not affected by steam and are designed to operate in temperatures up to $250^{\circ} \mathrm{C}$. This leads to the need of comparison of those two types of sensors, since we know, what exactly optical sensors measure, but what eddy-current measure exactly on shrouded blade is a question. Eddy-current design is shown in the Figure 2
The signal from eddy-current is sensitive on position of magnet and coil inside the sensor and on size of shroud compared to the size of sensor. The shape of signal is very dependent on position of sensor over blade shroud and on rotational frequency. The signal from sensor is amplified (A in figure 1) and transferred to TTL digital puls (trigger $\mathrm{T}$ in figure 1). The measuring system with clock ( $\mathrm{S}$ in figure 1) measures time arrivals of edges of TTL pulses. The system accuracy is dependent on sampling frequency of measuring clock. For example, if the rotational frequency $f_{r}$ is $60 \mathrm{~Hz}$ and rotor diameter $d=2904 \mathrm{~mm}$, to see vibration of order $10^{-1} \mathrm{~mm}$ we would need sampling frequency about $f_{s}=5.5 \mathrm{MHz}$, to see vibration of order $10^{-2} \mathrm{~mm}$ frequency $f_{s}=55 \mathrm{MHz}$ and to see vibration of order $10^{-3} \mathrm{~mm}$ even $f_{s}=550 \mathrm{MHz}$. Longer blades need even higher sampling frequency, disk with diameter $5 \mathrm{~m}$ would have blade tip speed about $940 \mathrm{~m} / \mathrm{s}$, so to measure with $10^{-3} \mathrm{~mm}$ accuracy, frequency $f_{s}=940 \mathrm{MHz}$ would be needed. For this measurement the sampling frequency was $200 \mathrm{MHz}$, which provided accuracy about $3 \mu \mathrm{m}$.

\subsection{Measurement Setup}

We used optical sensor and two similar eddy-current sensors with slightly different properties (size, number of loops in coil). Those sensors were attached to a threaded rod with stepper motor, that allowed us to move those sensors along the blade shroud step by step to measure on different positions without requirement of running-down and moving the sensors manually (Figure 3).

We were able to chose the speed of sweep and position arbitrarily and we saved values of the positions so we could compare it with the measurement. By the optical sensor we measured both, leading and trailing edge of the blade (Figure 4), so we were able to capture the whole blade shroud shape. Results are summarized in next sections.

\section{Results}

We have done two types of measurement: First, we investigated the influence of magnet and coil position to blade and secondly we excited blades on its natural frequencies to compare amplitudes from different sensors. We didn't move the sensors continuously, but we stopped every one $\mathrm{mm}$ for a few seconds to capture the vibration on every position.

\subsection{Sensor Orientation}

We performed five measurements, where we measured the blade shroud with sensor moving there and back starting on trailing edge, so we were sure, that the captured shape is not affected by level of vibration or inaccurate movement of sensors. We chose sensor orientation as shown in Figure 5 where circle marks the coil in the sensor. Starting by positioning the coil towards the blade, next positions were determined by turning the sensor by $90^{\circ}, 180^{\circ}, 210^{\circ}$ and $300^{\circ}$. 


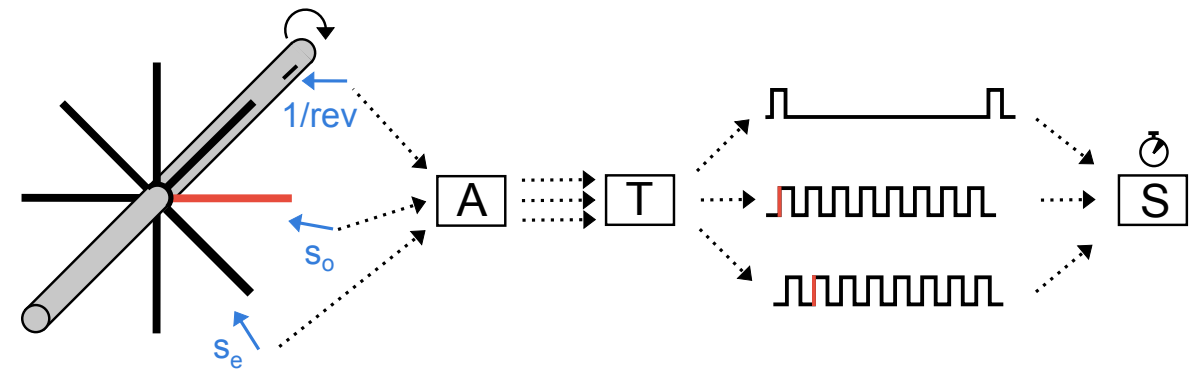

Figure 1. Diagram of BTT measuring system.

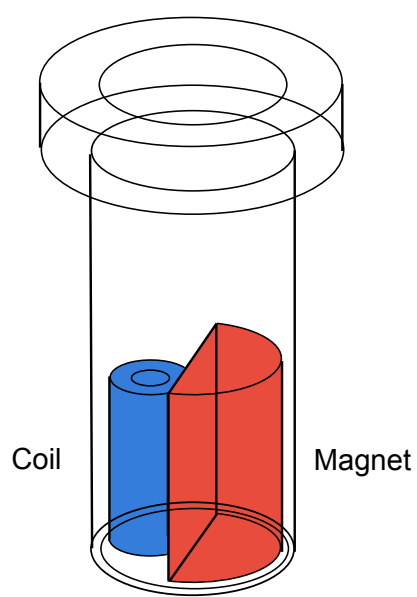

FiguRE 2. Eddy-current sensor.

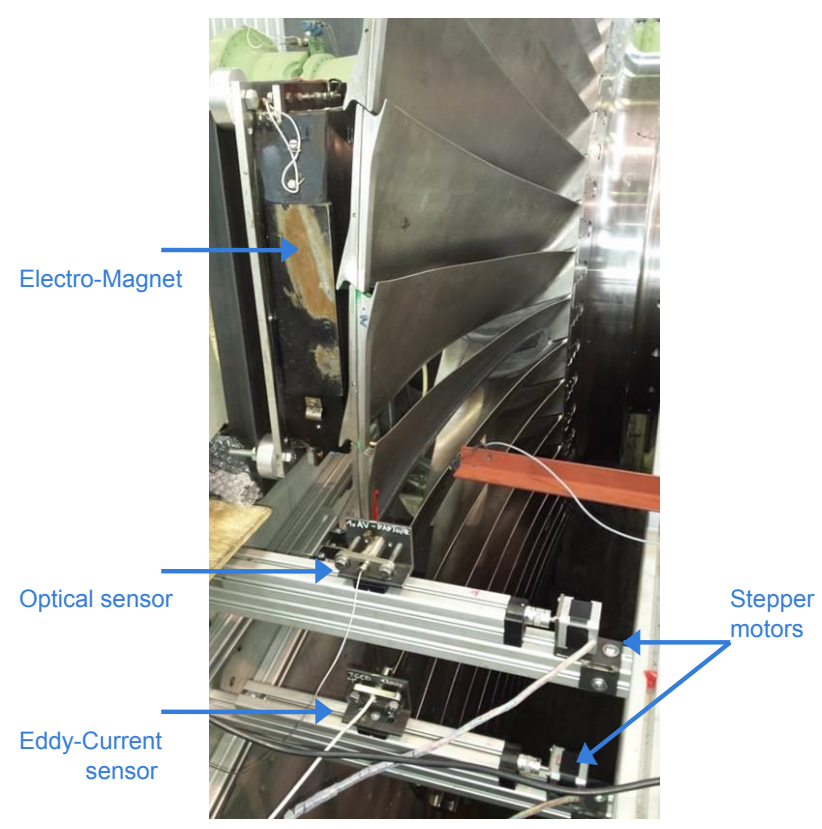

FiguRE 3. Measurement setup.

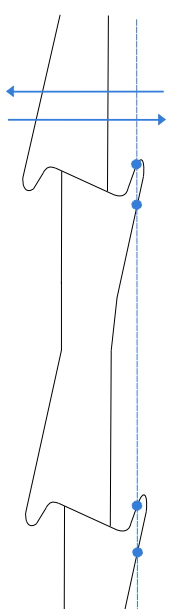

Figure 4. Edges captured by optical sensor.

To ilustrate the result we chose optical sensor and one eddy-current. Those sensors centers were distant from each other $190 \mathrm{~mm}$ (1.04 blade distance) and the data form each sensor are related to the $1 / \mathrm{rev}$ sensor. If we choose one specific blade, for example the first one that arrives to optical sensor after $1 / \mathrm{rev}$ mark, it is actually the second one, that arrives to eddy-current. The position of $k$-th blade of sensor $s$ in relative angle $p_{k}$ in revolution $n$ can be expressed as

$$
p_{k, n}=\frac{T O A_{s_{k, n}}-T O A_{r_{n}}}{T O A_{r_{n+1}}-T O A_{r_{n}}},
$$

where TOA stands for Time Of Arrival, $r$ for sensor $1 /$ rev and $s$ for optical sensor, resp. eddy-current. We take $k$-th blade from optical sensor and $k+1$-th blade from eddy-current, recalculate relative angle to $\mathrm{mm}$, subtract the distance of sensors and the result is shown in Figure 6. Data from eddy-currents are displayed as colored dots, colors correspond to orientation of sensor from Figure 5. The shape of blade shroud is taken from the optical sensor data. TE in the Figure6 stands for trailing edge and LE for leading edge.

The sensor size is for this blade comparable with the size of the blade shroud, what is in our opinion the cause of some uncertainty of what eddy-current measures, especially on leading edge. From experience it is expected, that the sensor measures by the center of the coil, which unfortunately wasn't confirmed by this measurement. This measurement was very important, because it confirmed, that on trailing edge is the 


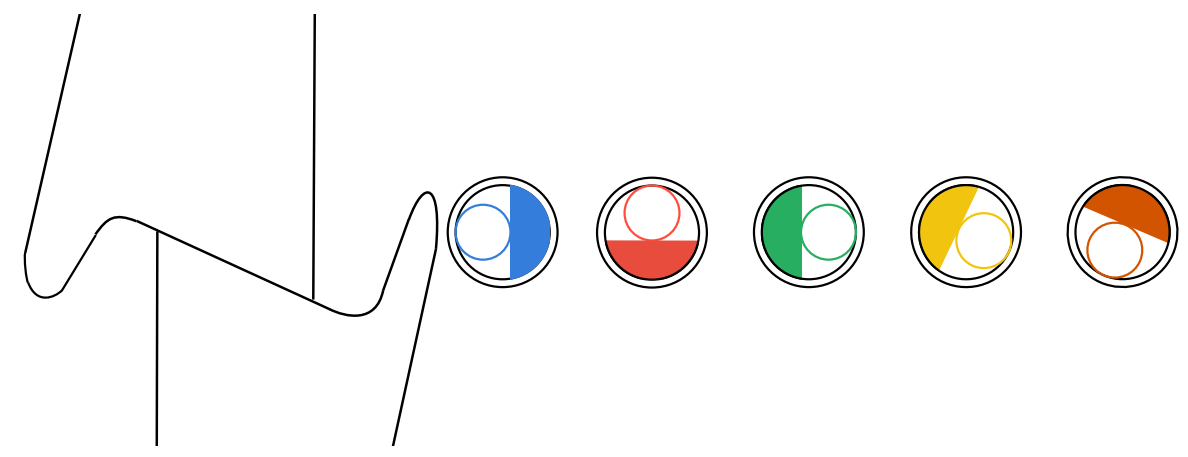

Figure 5. Eddy-current sensor orientation.

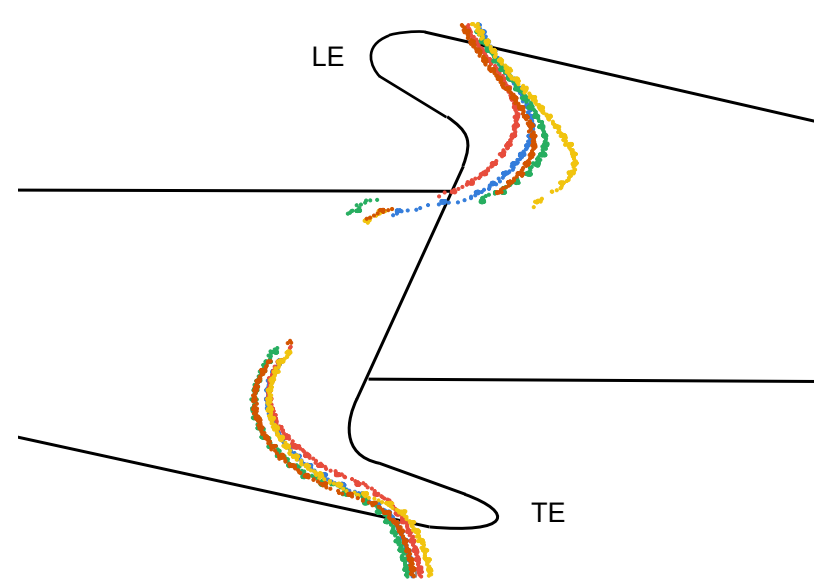

FiguRE 6. Eddy-current sensor data (Relative angle position of one blade in disk.)

measurement almost independent on orientation and that here it captures the blade shroud shape appropriately (even if it may not be exactly on the edge). Trailing edge is just the position, that is mainly used for BTT measurement. More in section 4

\subsection{Amplitude on NATURAL FREQUENCy}

Another measurement we performed, was exciting the bladed disk on its natural frequency and comparison of amplitude of optical and eddy-current sensors. We excited the blades on frequency $f_{e}$, see equation 1 . where the exact natural frequency $f_{n}$ was determined from strain gages measurement. We again measured the blade shroud there and back with few seconds stops, so we were able to use FFT on data in one position with sufficient accuracy. To compare amplitude form both sensors we used amplitude from all-blade spectrum. From parts of the data, where the sensors were steady, the all-blade FFT was computed. The amplitude on excitation frequency $f_{e}$ from this spectrum was taken and that is represented by color in the Figure 7. In the Figure 7 are results for two natural frequencies, $0 \mathrm{ND}$ and $6 \mathrm{ND}$.

As mentioned in section 2, we use torsional shaking for excitation of 0ND and electro-magnet for higher nodal diameters. From the Figure 7 0ND results we can see, that on trailing edge both sensors measure
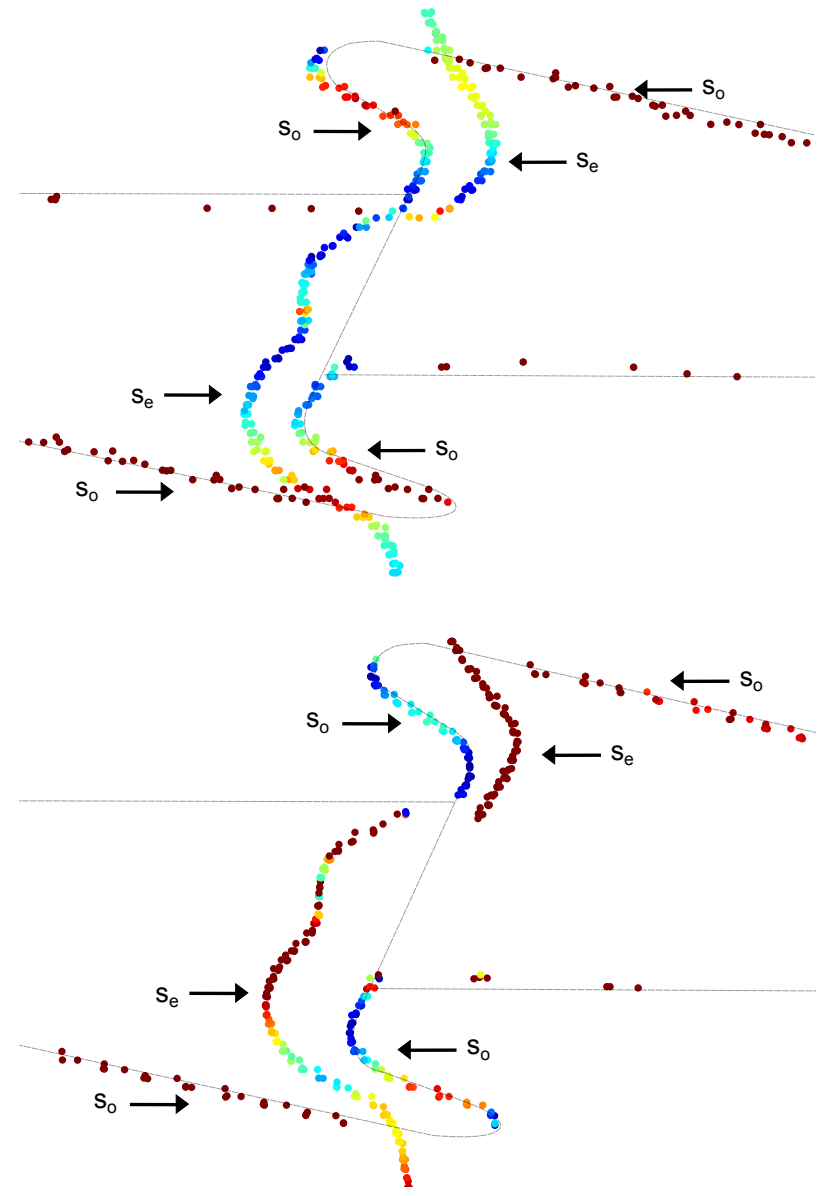

Figure 7. Amplitude for optical and eddy-current sensors on 0ND (top) and 6ND (bottom). $s_{o}$ - optical sensor, $s_{e}$ - eddy-current.

the same amplitude (color of eddy-current and optical sensor matches). That shows us the optimal position for measuring this blade. Unfortunately, as we can see in results for $6 \mathrm{ND}$, eddy-current sensor measurement was affected by the electro-magnet excitation increasingly, as the sensor was getting closer to the electro-magnet, that was placed on the leading edge side of the blade, see Figure 3 , where its position is shown. For higher nodal diameters only data from optical sensor from this measurement were reliable. 


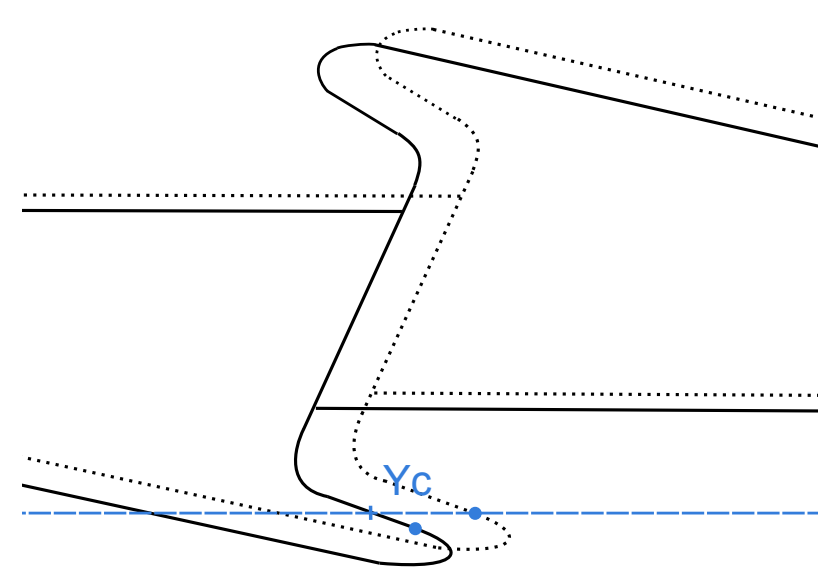

Figure 8. Deflection measured by BTT.

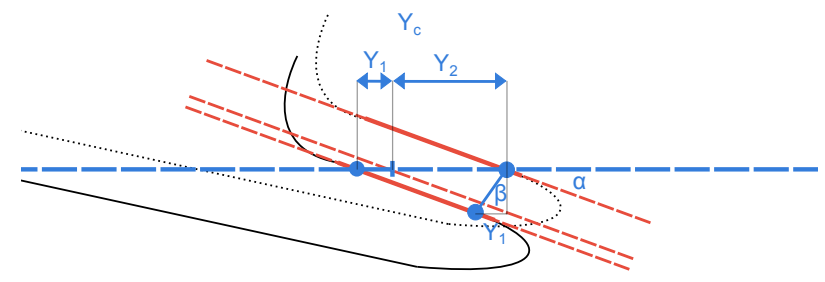

FiguRE 9. Recalculation of deflection measured by BTT to the actual deflection.

\section{Calibration Method}

Let us conclude with a description of how we use the results we get from this measurement and how we compare it with calculation.

The BTT sensor measures deflection in the direction of rotation, in the Figures 8 and 9 marked as $Y_{c}$, however the actual vibration of blade in this direction is $Y_{1}$.

Using angles $\alpha$ and $\beta$ we have

$$
Y_{c}=\left(\frac{1}{\tan \alpha \tan \beta}+1\right) Y_{1} .
$$

From here comes the importance of angles $\alpha$ and $\beta$. The angle $\alpha$ is given by blade design and angle $\beta$ by mode shape - is different for every nodal diameter and is computed by FEM.

If we take measurement from strain gages in $\mu \mathrm{m} / \mathrm{m}$ and divide it by actual amplitude measured by BTT, we get a ratio that expresses strain on one mm of blade tip deflection for specific strain gage position. This can be also calculated using FEM and those two values we compare to verify computed blade properties.

\section{Conclusion}

The measurement brought a new look on measuring with eddy-current sensor and we found an optimal position of sensor over blade shroud for this type of blade. The results may be extended to graphical comparison of measured data to FEM analysis depending on nodal diameter, type of sensor etc. To get better results with excitation higher nodal diameters using eddy-current sensors it is necessary to find a way of exciting the blades without affecting the sensor signal.

We expect a repetition of this measurement in the test rig with another type of blade with larger diameter. This could demonstrate the difference of sensor signal behaviour, especially on leading edge, where the measuring point of eddy-current for this blade is unclear.

\section{LIST OF SYMBOLS}

$f_{s} \quad$ Sampeling frequency of measuring clock $[\mathrm{Hz}]$

$f_{e} \quad$ Excitation frequency $[\mathrm{Hz}]$

$f_{n}$ Natural frequency $[\mathrm{Hz}]$

$f_{r} \quad$ Rotational frequency $[\mathrm{Hz}]$

$N D$ Nodal Diameter

$p_{k, n} \quad$ Relative angle of $\mathrm{k}$-th blade in $\mathrm{n}$-th revolution

$T O A_{s_{k, n}}$ Time of arrival of k-th blade in $\mathrm{n}$-th revolution to sensor s

TOA $A_{r_{n}}$ Time of arrival of $1 /$ rev marker in $\mathrm{n}$-th revolution

$Y_{c} \quad$ Displacement seen by BTT $[\mathrm{mm}]$

$Y_{1}$ Actual displacement of blade in tangential direction $[\mathrm{mm}]$

\section{ACKNOWLEDGEMENTS}

These research's results are part of the TURBO-REFLEX project, which has received funding from the European Union's Horizon 2020 research and innovation programme under grant agreement No 764545 .

\section{REFERENCES}

[1] Z. Kubin, J. Liska, T. Misek, P. Prochazka. Application of non-contact vibration measurements on a 48 " blade under low load condition. DYMAMESI 2017 Prague: Institute of Thermomechanics AS CR pp. 45-54, 2017.

[2] G. Battiato, C. Firrone, T. Berruti. Forced response of rotating bladed disks: Blade tip-timing measurements. Mechanical Systems and Signal Processing 85(1):912-926, 2017. DOI:10.1016/j.ymssp.2016.09.019.

[3] O. Danek, F. Vanek, J. Kozanek, et al. The development of identification of dynamic parametres of rotating machinery parts. Proceedings of the conf Structural system Identification 2001.

[4] P. Prochazka, F. Vanek. New methods of non-contact sensing of blade vibrations and deflections in turbomachinery. IEEE Trans Instrum Meas 63:1583-1592, 2014

[5] N. Jamia, M. Friswell, S. El-Borgi, R. Fernandes. Simulating eddy current sensor outputs for blade tip timing. Advances in Mechanical Engineering 10(1), 2018.

[6] Z. Kubin, T. Misek, J. Hlous, et al. Calibration of blade tip-timing sensor for shrouded 40" last stage blade. Mechanical Systems and Signal Processing 108:88-98, 2018. DOI:10.1016/j.ymssp.2018.02.001 